\title{
Global minimization in few-body systems
}

C. Voglis ${ }^{\mathrm{a}}$, I.E. Lagaris ${ }^{\mathrm{a}, \mathrm{b}}$, M.L. Lekala ${ }^{\mathrm{b}}$, G.J. Rampho ${ }^{\mathrm{b}}$, S.A. Sofianos ${ }^{\mathrm{b}}$.

a Department of Computer Science, University of Ioannina, GR 451 10, Ioannina, Greece

${ }^{\mathrm{b}}$ Physics Department, University of South Africa, Pretoria 0003, South Africa

The use of global optimization (GO) techniques in few-body systems is discussed. Their usefulness is demonstrated by applying them to solve the two-body bound state problem by casting the wave function as an artificial neural network (ANN) as well as by employing them to locate zeros of the Jost function in the complex $k$-plane corresponding to bound and resonance states.

\section{INTRODUCTION}

Optimization techniques have been extensively used in a variety of physical problems in Many-Body systems such as the construction of optical potentials, phase shift analyses, variational calculations, neural network training, etc. In the field of Few-Body systems however, their use is rather limited although there are cases where the method could be very useful. A subtle feature is that when the original problem is transformed to an optimization one, the resulting objective function possesses a host of local minima. From these minima only a few may correspond to physically acceptable solutions, hence local optimization is inadequate and GO methods are required.

A typical example is the evaluation of the spectrum of a heavy three-molecular system using Faddeev-type methods where one has to evaluate the plethora of low lying states $E_{\nu}$, $\nu=1,2, \cdots, N$ in a relatively small energy window $\Delta E$. The spectrum is usually obtained using standard few-body methods based on finite element techniques the accuracy of which depends on the polynomial chosen, the distribution and density of the relevant grid points employed, and the handling of the resulting huge matrices. The calculations are often tedious and in certain cases problematic as the calculated spectrum depends on the total accuracy of solving the three-body equations and each individual evaluation of a spectral point, results in a binding energy that lies in an energy range where several other binding energies exist and therefore it is not readily clear for which $\nu$ the binding energy found corresponds. The problem becomes even more complex when one wants to construct or improve three-molecular forces under the constrain that these should reproduce the lowlying spectrum of the system. These forces should complement the highly realistic and well known interatomic interactions and a fine tuning to evaluate the parameters of the three-body force so that the low lying states are simultaneously reproduced is desirable. In such cases the use of a GO is necessary.

Yet another characteristic example is the location of the zeros of the Jost function 
$f_{\ell}(k)$ on the entire complex $k$-plane. The potential resonances, for example, correspond to the zeros of the Jost function in the first quadrant of the $k$-plane. This is a quite time consuming procedure especially when narrow resonances are present or when the complete resonances trajectories are required.

It should be noted that minimizing a multidimensional function entails a lot of inherent difficulties. There is not a single method that can tackle all problems in a satisfactory way and one needs a strategy, combining different methods, to efficiently handle a wide spectrum of problems. MERLIN is an integrated environment designed to solve optimization problems [1]. It is devised to be easy-to-use, and implemented so as to be portable among different platforms. The GO method used [2], is of stochastic nature and attempts to recover all the local minima of an objective function inside a rectangular domain ${ }^{1}$.

\section{APPLICATIONS}

\subsection{Binding Energies with Neural Networks}

There is a plethora of methods to solve the two-body Schrödinger equation

$\left[\frac{\mathrm{d}^{2}}{\mathrm{~d} r^{2}}+k^{2}-\frac{\ell(\ell+1)}{r^{2}}-\frac{2 \eta k}{r}\right] \Phi_{\ell}(\eta, k, r)=V(r) \Phi_{\ell}(\eta, k, r)$

Some of the methods are based on techniques that use an expansion of the wave function $\Phi_{\ell}(\eta, k, r)$ in terms of polynomials, such as the piecewise Hermite and B-splines polynomials, the Chebyshev polynomials, etc., and thus these methods can be readily extended to multidimensional cases as well. The expansion parameters appear in a linear way and therefore one has to use a large number of terms to achieve high accuracy and this, in turn, necessitates the handling of huge matrices when one goes beyond the single-variable two-body problem.

In our approach we employ, following [3], ANNs to achieve a more economical representation of the solution. However the resulting problem is non-linear, and therefore the use of sophisticate optimization techniques is necessary. The ANN used is of the form

$N(\vec{a}, \vec{b}, \vec{c}, r)=\sum_{i=1}^{N} \frac{a_{i}}{1+\exp \left(-b_{i} r+c_{i}\right)}$.

We demonstrate the method using the molecular potential

$V(r)=5 \exp \left[-0.25(r-3.5)^{2}\right]-8 \exp \left(-0.2 r^{2}\right)$.

where the strengths are given in $\mathrm{MeV}$ and the distances in fm with $\hbar^{2} / 2 m=1 / 2 \mathrm{MeV} \mathrm{fm}$. In the absence of Coulomb forces $(\eta=0)$ we obtain, with $N=5$, the energy eigenvaluesvalues 3.023634509 and 1.329872790 that differ in the 9 th significant digit from those found in [5].

\subsection{Potential Resonances}

To demonstrate the application of the GMM we apply it to locate potential resonances for central potentials $V(r)$ with $\lim _{r \rightarrow 0} r^{2} V(r)=0, \quad \lim _{r \rightarrow \infty} r V(r)=0$. Following the

\footnotetext{
${ }^{1}$ One may download the programs from the site: http://nrt.cs.uoi.gr/merlin/
} 
formalism of $[4,5]$ and by considering the complex rotation

$$
r=x \exp (i \theta), \quad x \geq 0, \quad \theta \in\left[0, \theta_{\max }\right], \quad \theta_{\max }<\pi / 2,
$$

we may look for physical solution of (1) in the form

$\Phi_{\ell}\left(\eta, k, x \mathrm{e}^{i \theta}\right)=\frac{1}{2}\left[H_{\ell}^{(+)}\left(\eta, k x \mathrm{e}^{i \theta}\right) \mathcal{F}_{\ell}^{(+)}(\eta, k, x, \theta)+H_{\ell}^{(-)}\left(\eta, k x \mathrm{e}^{i \theta}\right) \mathcal{F}_{\ell}^{(-)}(\eta, k, x, \theta)\right]$

where $H_{\ell}^{( \pm)}$are combinations of the regular and irregular Coulomb functions $H_{\ell}^{( \pm)}(\eta, z) \equiv$ $F_{\ell}(\eta, z) \mp i G_{\ell}(\eta, z)$ while the $\mathcal{F}_{\ell}^{( \pm)}(k, z)$ obey the system of first order differential equations

$$
\begin{aligned}
\frac{\mathrm{d}}{\mathrm{d} x} \mathcal{F}_{\ell}^{(+)}(\eta, k, x, \theta) & =\frac{\mathrm{e}^{i \theta}}{2 i k} H_{\ell}^{(-)}(\eta, k, x, \theta) V\left(x \mathrm{e}^{i \theta}\right) \\
& \times\left[H_{\ell}^{(+)}\left(\eta, k x \mathrm{e}^{i \theta}\right) \mathcal{F}_{\ell}^{(+)}(\eta, k, x, \theta)+H_{\ell}^{(-)}\left(\eta, k x \mathrm{e}^{i \theta}\right) \mathcal{F}_{\ell}^{(-)}(\eta, k, x, \theta)\right] \\
\frac{\mathrm{d}}{\mathrm{d} x} \mathcal{F}_{\ell}^{(-)}(\eta, k, x, \theta) & =-\frac{\mathrm{e}^{i \theta}}{2 i k} H_{\ell}^{(+)}(\eta, k, x, \theta) V\left(x \mathrm{e}^{i \theta}\right) \\
& \times\left[H_{\ell}^{(-)}\left(\eta, k x \mathrm{e}^{i \theta}\right) \mathcal{F}_{\ell}^{(+)}(\eta, k, x, \theta)+H_{\ell}^{(-)}\left(\eta, k x \mathrm{e}^{i \theta}\right) \mathcal{F}_{\ell}^{(-)}(\eta, k, x, \theta)\right] .
\end{aligned}
$$

The corresponding Jost function $f_{\ell}(\eta, k)$ is then given by

$f_{\ell}(\eta, k) \underset{x \rightarrow 0}{\longrightarrow} \mathcal{F}_{\ell}^{(-)}(\eta, k, x, \theta)$

The required boundary conditions for the solution of the system (5-6) are discussed in $[4,5]$. We recall here the simple condition at origin

$\mathcal{F}_{\ell}^{(+)}(\eta, k, 0, \theta)=\mathcal{F}_{\ell}^{(-)}(\eta, k, 0, \theta)=1$.

The search for zeros of the Jost function, $f(k) \equiv f(a+i b)=0$ in the complex $k$-plane, as mentioned, is not an easy task. From the theory of scattering we have the following physical properties of $f(k)$,

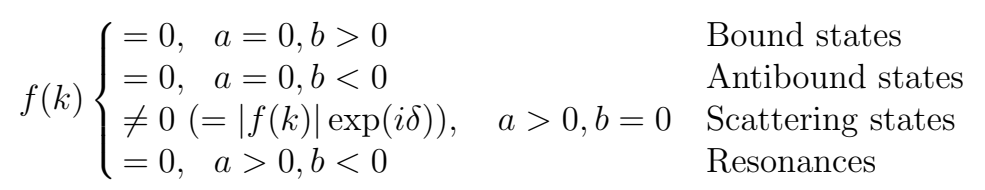

Thus, after complex rotation, and the solution of the system (5-6), we may extract $f(k)$. The accurate solution of the differential equations is crucial in obtaining the $f(k)$. For this we employed the differential equation solver of Shampine and Gordon [6] in which one may achieve an accuracy up to the machine's constant.

Using the above potential and GO we obtain the results shown in table 1 . The accuracy depends on the tolerance chosen for solving the differential equations to obtain the Jost function. We choose in the present case $\epsilon=10^{-8}$ while the rotation angle is chosen to be $\theta=22$ degrees. The results are then readily obtained within a minute or so on a PentiumIV CPU running at $2.0 \mathrm{GHz}$. The time depends on the number of specified resonances to be found, the required accuracy and the available computer power. We must emphasize here that the angle $\theta$ should be greater than the angle $\varphi$ that defines the position of the resonance $k_{r}=\left|k_{r}\right| \exp (-i \varphi)$. In such a case the function $\Phi_{\ell}(\eta, k, x \exp [i \theta])$ is square integrable. From this table we see that the reproduction of the bound states (upper two lines) and of the resonances is excellent. 
Table 1

Bound states and resonances obtained with the potential (3).

\begin{tabular}{cc}
\hline$k\left(\mathrm{fm}^{-1}\right)$ & GMM \\
\hline $0.000000000+i 3.023634507$ & $0.000000000+i 3.023634572$ \\
$0.000000000+i 1.329872758$ & $0.000000000+i 1.329872751$ \\
\hline $2.122442334-i 0.000027859$ & $2.122442334-i 0.000027859$ \\
$3.000600515-i 0.041316851$ & $3.000600511-i 0.041131670$ \\
$3.485234669-i 0.360967988$ & $3.485234667-i 0.360967968$ \\
$3.974580284-i 0.788297975$ & $3.974580276-i 0.788297981$ \\
$4.454733926-i 1.227097054$ & $4.454733890-i 1.227097080$ \\
$4.922800349-i 1.664647328$ & $4.922800556-i 1.664737506$ \\
$5.378677040-i 2.097566794$ & $5.378677063-i 2.097566507$ \\
\hline
\end{tabular}

\section{CONCLUSIONS}

We have demonstrated that global optimization is a power tool which can be used to solve tough problems encountered in Few-Body systems. The available MERLIN and associated software in conjunction with the present day computing power are the ingredients that guarantee a successful use of GO in a variety of fields such as training of neurals, variational approaches, locating potential resonances, construction of two- and three-body forces and elsewhere.

\section{REFERENCES}

1. D.G. Papageorgiou, I.N. Demetropoulos and I.E. Lagaris, Comput. Phys. Commun. 159 (2004) 70.

2. D.G. Papageorgiou, F.V. Theos and I.E. Lagaris, Comput. Phys. Commun. 159 (2004) 63.

3. I.E. Lagaris, A. Ligas and D.I. Fotiadis, IEEE Transactions on Neural Networks 9 (1998) 981; Comput. Phys. Commun. 104 (1997) 1

4. S.A. Rakityansky, S.A. Sofianos and K. Amos, Il Nuovo Cimento, 111 B (1996) 353.

5. S.A. Sofianos and S.A. Rakityansky, J. of Phys. A 30 (1997) 3725.

6. L.F. Shampine and M.K. Gordon, Computer Solution of Ordinary Differential Equations: The Initial Value Problem WH Freeman, San Francisco 1975. 